Palaeohispanica 2, (2002), pp. 411-414

\title{
CHRONICA EPIGRAPHICA IBERICA IV (2001)
}

Javier Velaza

1. Nueva leyenda monetal de Saitabi.

P.P.Ripollès Alegre, "Una leyenda monetal inédita de Saitabi", Saguntum 33 (2001), pp. 167-170.

El autor edita un didracma de plata de la ceca de Saitabi a la que ha tenido acceso a través de una impronta realizada hace unos quince años por Alberto Canto. Su peso es desconocido y su descripción es la siguiente: "Anv. Cabeza de Herakles con leonté, a dcha.; no lleva una clava sobre el hombro izq. Gráfila de puntos. Rev. Águila con alas abiertas a dcha.: por detrás y por encima de ella, inscripción ibérica. Gráfila de puntos".

\section{śaitabikitarban}
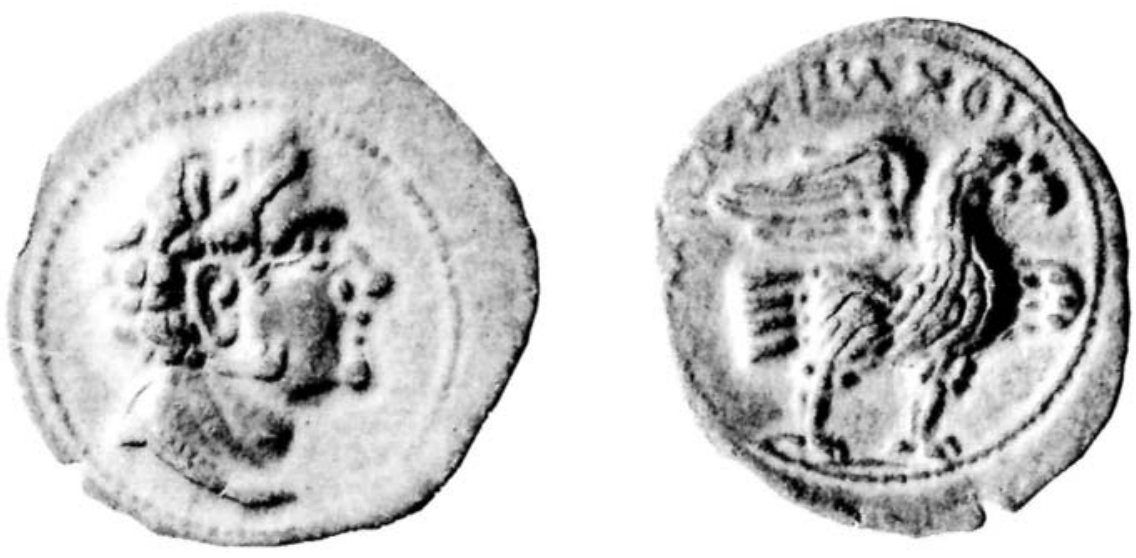

Didracma con la leyenda śaitabikitarban (fotografía P.P. Ripollès) 
La leyenda era inédita hasta el momento, puesto que la única pieza que se conocía de la emisión, aparecido en el tesoro de Valeria, sólo permitía leer los dos primeros signos. Para su interpretación, conviene seguramente apelar a la semejanza con la leyenda monetal saguntina arskitar, en la que se detecta la misma estructura constituida por el radical toponímico y la forma kitar. En la didracma de Saetabi, a diferencia de la de arse, la secuencia aparece seguida del elemento ban que, como se sabe, es también frecuente en la epigrafía monetal.

2. Plomo procedente del Camp de Morvedre.

L.Silgo Gauche-A.Tolosa Leal, "Plomo ibérico escrito del Camp de Morvedre", Arse 34 (2000), pp. 39-44.

Lámina de plomo de forma semicircular $(3,9 \times 4,2 \mathrm{~cm}$, medidas máximas) y grosor irregular (entre 0,5 y $0,25 \mathrm{~cm}$ ), a lo que parece incompleto y notablemente desgastado en su superficie. Presenta inscripción en ambas caras, en signario levantino y probablemente producto de manos distintas. Procedería de un hallazgo casual en la comarca del Camp de Morvedre, según informan los editores, quienes sólo tuvieron una fugaz ocasión de estudiarlo y obtener fotografías. La lectura propuesta por los editores es la siguiente.

a)

$$
\begin{aligned}
& \text {---]*rba : nkearsboiltiŕ : baitibo**'ŕ[--- } \\
& \text {---]i[---]n[--- }
\end{aligned}
$$

b)

$$
\begin{aligned}
& \text { etemiltió : baiesatababeŕ } \\
& \text { arkio }
\end{aligned}
$$
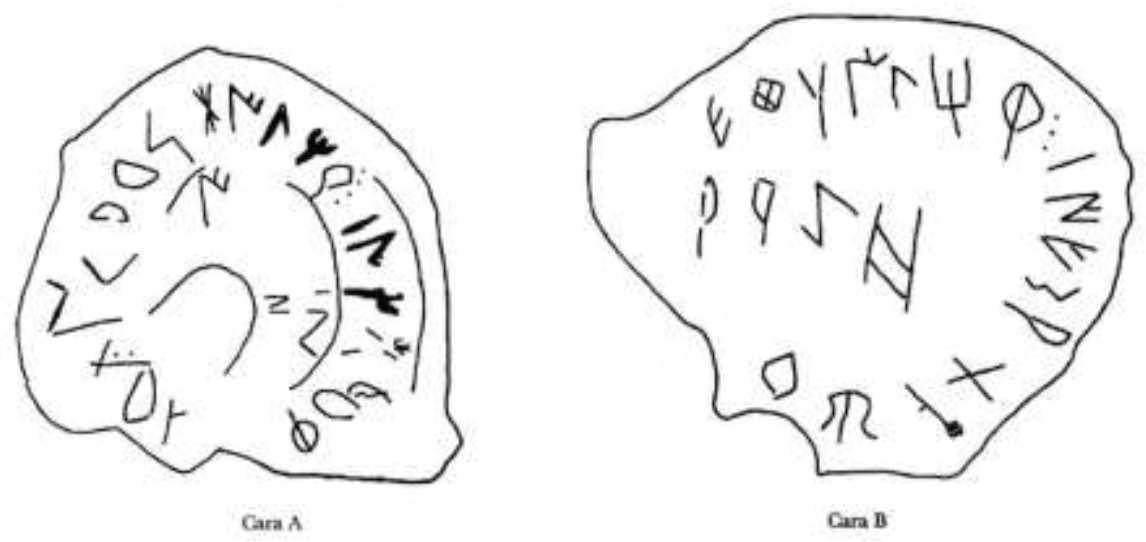

Plomo de Camp de Morvedre (dibujo Silgo-Tolosa) 


\section{Chronica epigraphica iberica IV (2001)}

Es muy difícil pronunciarse sobre diferentes aspectos de esta inscripción sin posibilidad de autopsia y contando solamente con las fotografías publicadas. Los editores, por ejemplo, no parecen cuestionarse en ningún momento su autenticidad, quizás porque la autopsia les ha proporcionado evidencias suficientes de ella. En otro orden de cosas, la lectura es dudosa en varias partes. En el texto a), por ejemplo, se pueden ver rastros de signos sin identificar, y no es seguro el sentido de la lectura, que podría también comenzar por arsboiltión. El texto b) resulta menos conflictivo en cuanto a la identificación de los signos.

Desde el punto de vista lingüístico, el único elemento con buenos paralelos léxicos es iltiŕ, repetido en ambas caras.

3. Nueva interpretación de la inscripción pintada sobre cerámica de Torrelló del Boverot (Almazora, Castellón).

J. Rodríguez Ramos, "Sobre los signos de lectura problemática en la escritura ibérica levantina y una inscripción revisada", AespA 74 (2001), pp. 281-290.

El autor propone una nueva interpretación del texto editado por G.Clausell-I.Izquierdo-F.Arasa, "La fase del Ibérico final en el asentamiento del Torrelló del Boverot (Almazora, Castellón): dos piezas cerámicas singulares", AespA 73 (2000), pp. 87-104 y recogido en J.Velaza, "Chronica epigraphica Iberica III (2000)", Palaeohispanica 1 (2001), pp. 394-395, n. 2. La hipótesis de lectura de Rodríguez Ramos, que se basa en considerar que el texto ha sido pintado de derecha a izquierda, es la siguiente:

\section{$[---] a$ : ultiba*[---]}

Para el primer signo se propone alternativamente $\underline{\mathbf{r}}$. La hipótesis parece atractiva, a pesar de que los epígrafes de orientación sinistrorsa son muy inhabituales en la epigrafía ibérica. La propuesta solucionaría el problema paleográfico del signo 4 , y permitiría identificar, por otra parte, un segmento ulti- frecuente en el léxico como formador de nombres personales.

\section{Ponderal de Calafell.}

J.Sanmartí-J.Velaza, "Un ponderal amb inscripció ibèrica de Calafell", Fonaments, en prensa.

Se trata de un ponderal de arcilla cuyas dimensiones son $9 \times 11,3 \times$ 6,25. Los datos procedentes del contexto arqueológico permiten establecer un terminus ante quem para su uso en torno al $200 \mathrm{aC}$. Presenta un orificio que lo atraviesa. En una cara de las caras laterales hay un signo cruciforme grabado antes de la cocción de la pieza. En la cara anterior y en sentido horizontal se ha esgrafiado con un instrumento de punta seca el texto de la inscripción, que consta de tres líneas. La primera comienza en el ángulo superior izquierdo y corre paralela al borde superior hasta el ángulo derecho, donde la línea de escritura gira para seguir luego parale- 
la al borde derecho del ponderal. Los signos presentan un módulo desigual, que va desde los $2,7 \mathrm{~cm}$ del primero de 1.1 (en el que se advierte, de todos modos, una clara voluntad de "mayusculizarlo") hasta los 1,1 $\mathrm{cm}$ del último signo de I. 3. La tipología paleográfica es más bien arcaizante (a1, e1, i1, o3, u3, be3, ke10, ki6, te14, ti7/ti1, n1, 12, r3, ŕ7, s3, ś1).

\section{akirukeŕte : iustir baśbeŕ akiroŕtin ultilatie}

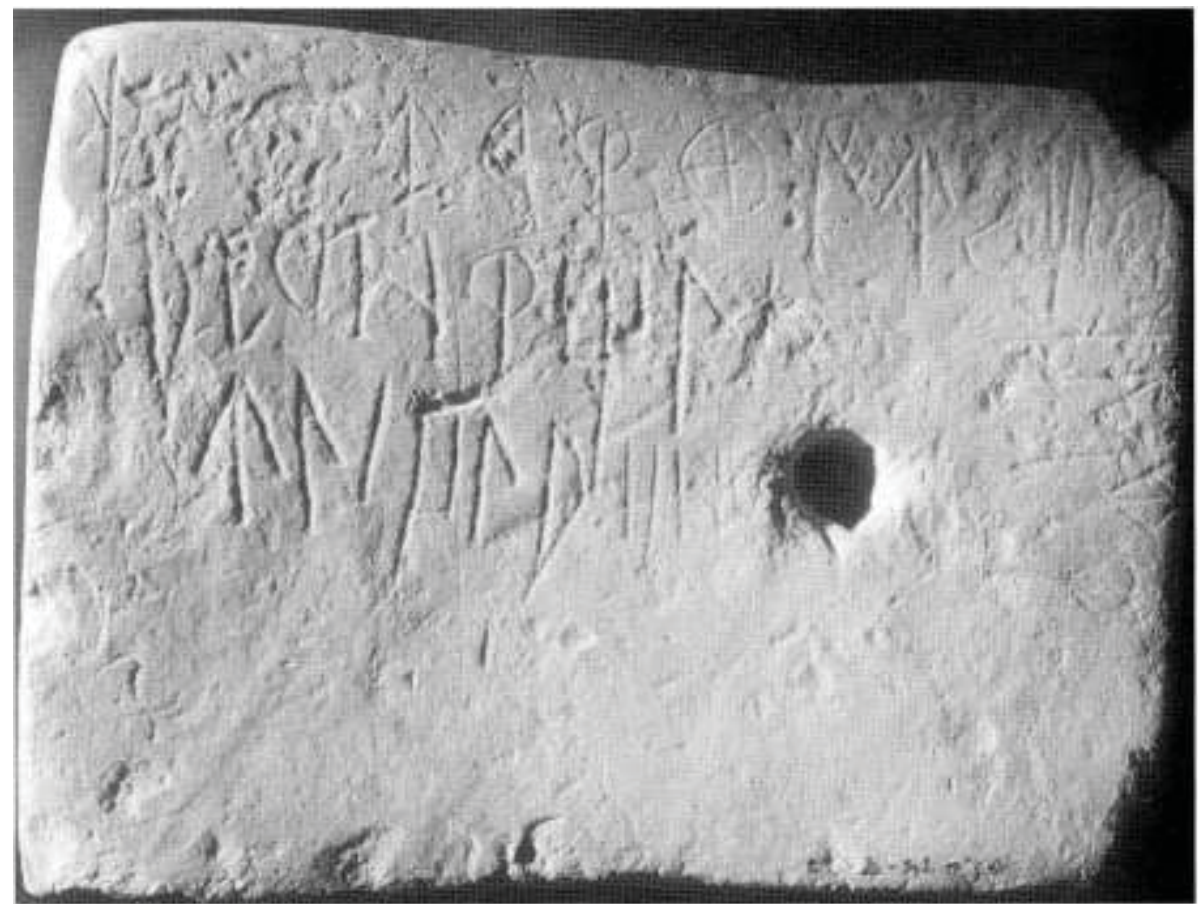

Ponderal de Calafell (fotografía J. Sanmartí)

Para un estudio pormenorizado de las palabras y la estructura del texto, remitimos a la edición arriba señalada. En síntesis, pueden identificarse tres nombres de persona, akirukér, akiroŕtin y ultilati En la primera línea, además, parece que la secuencia es NP-te iustir, lo que, a juzgar por el paralelo con NP-te ekiar, invitaría a adjudicar a iustir un valor verbal (o cuasi-verbal). 